OPEN ACCESS

Edited by:

Huilin $\mathrm{Hou}$

Ningbo University of Technology,

China

Reviewed by:

Nan Zhu,

Dalian University of Technology, China

${ }^{*}$ Correspondence:

Saisai Yuan

yuansaisai66666@163.com

Qitao Zhang

qitao-zhang@szu.edu.cn

Specialty section:

This article was submitted to Catalysis and Photocatalysis, a section of the journal

Frontiers in Chemistry

Received: 10 November 2021 Accepted: 26 November 2021 Published: 17 December 2021

Citation:

Yuan S and Zhang Q (2021) Application of One-Dimensional Nanomaterials in Catalysis at the

Single-Molecule and Single-

Particle Scale.

Front. Chem. 9:812287.

doi: 10.3389/fchem.2021.812287

\section{Application of One-Dimensional Nanomaterials in Catalysis at the Single-Molecule and Single-Particle Scale}

\author{
Saisai Yuan ${ }^{1 *}$ and Qitao Zhang $^{2 *}$ \\ ${ }^{1}$ School of Environmental and Chemical Engineering, Jiangsu University of Science and Technology, Zhenjiang, China, \\ ${ }^{2}$ International Collaborative Laboratory of 2D Materials for Optoelectronics Science and Technology of Ministry of Education, \\ Institute of Microscale Optoelectronics, Shenzhen University, Shenzhen, China
}

The morphology of nanomaterials has a great influence on the catalytic performance. Onedimensional (1D) nanomaterials have been widely used in the field of catalysis due to their unique linear morphology with large specific surface area, high electron-hole separation efficiency, strong light absorption capacity, plentiful exposed active sites, and so on. In this review, we summarized the recent progress of $1 \mathrm{D}$ nanomaterials by focusing on the applications in photocatalysis and electrocatalysis. We highlighted the advanced characterization techniques, such as scanning tunneling microscopy (STM), atomic force microscopy (AFM), surface photovoltage microscopy (SPVM), single-molecule fluorescence microscopy (SMFM), and a variety of combined characterization methods, which have been used to identify the catalytic action of active sites and reveal the mechanism of $1 \mathrm{D}$ nanomaterials. Finally, the challenges and future directions of the research on the catalytic mechanism of single-particle 1D nanomaterials are prospected. To our best knowledge, there is no review on the application of singlemolecule or single-particle characterization technology to 1D nanomaterial catalysis at present. This review provides a systematic introduction to the frontier field and opens the way for the 1D nanomaterial catalysis.

Keywords: 1D nanomaterials, photocatalysis, electrocatalysis, single-particle, single-molecule

\section{INTRODUCTION}

One-dimensional (1D) nanomaterial is a special structure of substance in nanometer scale and is a key branch of nanomaterial systems. It has been widely developed and been used in many fields due to its unique linear morphology with large specific surface area, high electron-hole separation efficiency, strong light absorption capacity, plentiful exposed active sites, and other characteristics (Linares et al., 2014). In general, 1D nanostructures have a high aspect ratio, with the diameters ranging from 1 to $100 \mathrm{~nm}$ (Xia et al., 2003), and their morphologies can be wired (Tian et al., 2007), tubular (Patil et al., 2017), rod-like (Liu et al., 2016a), fibrous (Hou et al., 2016), or banded (Sun et al., 2016). The preparation of 1D nanostructures mainly includes hydrothermal method (Byrappa and Adschiri, 2007), vapor deposition method (George, 2010), sol-gel method (Sui and Charpentier, 2012), template method (Ghahremaninezhad and Dolati, 2009), and electrospinning method (Li and Xia, 2004). Therefore, based on the characteristics of 1D nanomaterials, it has significant advantages in light capture, electron and ion transmission, 


\section{A}

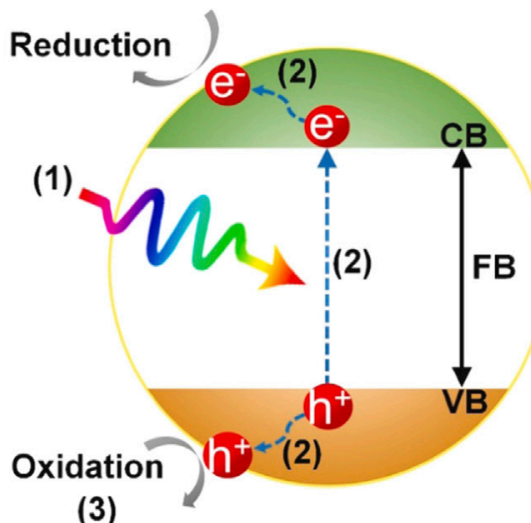

B

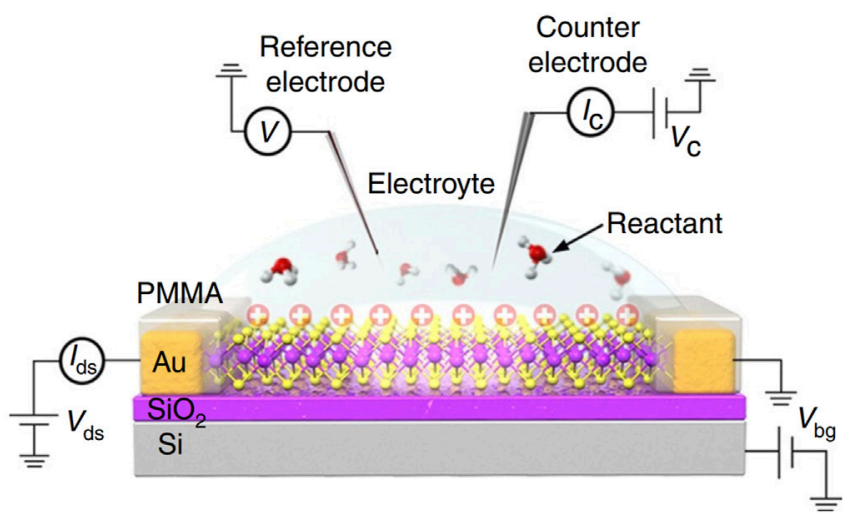

FIGURE 1 | Schematic diagram of the principles of photocatalysis and electrocatalysis. (A) The typical photocatalytic process goes through three steps (Zhu et al., 2021). (B) Schematic diagram of electrocatalytic measurement (He et al., 2019). Reproduced from Zhu et al. (2021) with the permission of the Elsevier. Reproduced from He et al. (2019) with the permission of Springer Nature.

and mass loading and diffusion and has an important application prospect in the field of mesoscopic, energy storage and conversion (Mai et al., 2014; Cao et al., 2019), nano-optoelectronic devices ( $\mathrm{Li}$ et al., 2013), photocatalysis (Liu et al., 2010), and electrocatalysis (Gao et al., 2018).

Among these applications, photocatalysis and electrocatalysis are of great interest to us in this review. Heterogeneous surface catalysis had been used in various catalytic reactions and had led to extensive exploration of the catalytic activity of metallic and nonmetallic surface sites (Wang et al., 2014; Low et al., 2017). The principles of photocatalysis are shown in Figure 1A, and the charge generated by light needed to be effectively separated from the surface of the photocatalytic materials for the catalytic reaction. The catalytic efficiency mainly depended on the charge separation (Zhang et al., 2018), light absorption capacity (Yuan et al., 2017; Yuán et al., 2018), and specific surface area (Yuan et al., 2014; Yuan et al., 2018) of the catalyst. Fast charge separation and slow recombination were beneficial to the formation of more carriers on the catalyst surface and excellent catalytic performance. At the same time, the response to visible light could be enhanced by tailoring the bandgap structure of the semiconductor, which generated more photogenerated charge pairs and further improved the rate of photocatalysts. For electrocatalysis, as shown in Figure 1B, it was a catalysis that accelerates the charge transfer at the interface between electrodes and electrolytes by external applied voltage. The understanding of the reaction mechanism of electrocatalysis is important for the design and development of electrocatalytic materials. The electrocatalytic performance was affected by the structure-activity relationship, the distribution of active sites, and the surface process of electrocatalytic reaction.

No matter in the field of photocatalysis or electrocatalysis, because of the complexity of the catalytic system, it is necessary to comprehensively characterize the catalytic process and deeply reveal the mechanism of the catalytic process, which is crucial to further develop the design of functional catalytic materials and further improve the catalytic performance. As we know, the catalytic reactions always take place on the surface of the catalyst, and the finer we disperse the same mass of material, the more atoms on the surface are exposed, resulting in the catalyst with a larger specific surface (Liu et al., 2016b; Chao et al., 2021). Reducing the size of the catalyst to a single particle can not only increase the effective utilization rate of the catalyst but also greatly improve its catalytic activity. In addition, compared with bulk catalysts, single-molecule or single-particle catalysts have a simple structure, have a clear definition of active sites, and avoid multi-pathway reactions in the catalytic process, which has a great advantage in the investigation of reaction mechanisms. Up to now, many surface characterization techniques have been used to investigate the catalytic process with good spatial, chemical, and time resolution and have made great contributions to the understanding of the catalytic process. However, these traditional characterization techniques only obtained the average information of many catalytic particles and aggregates. The morphology, structure, crystal plane, and composition of a single catalytic particle strongly affected the overall performance of the catalyst (Tachikawa et al., 2011). It is an ideal strategy to directly apply characterization techniques to the real complex surface catalytic systems, especially to study the surface structure of the catalyst and the surface process of the catalytic reaction at the single-molecule scale. The development of advanced techniques provides the opportunities to study the catalytic mechanism at singlemolecule and single-particle scale. As shown in Figure 2, scanning tunneling microscopy (STM), atomic force microscopy (AFM), surface photovoltage microscopy (SPVM), single-molecule fluorescence microscopy (SMFM), and so on, were of benefit to identify the catalytic action of active sites and reveal the mechanism of complex catalytic systems and further promote the design and development of novel catalysts (Novo et al., 2008; Tang et al., 2011). 


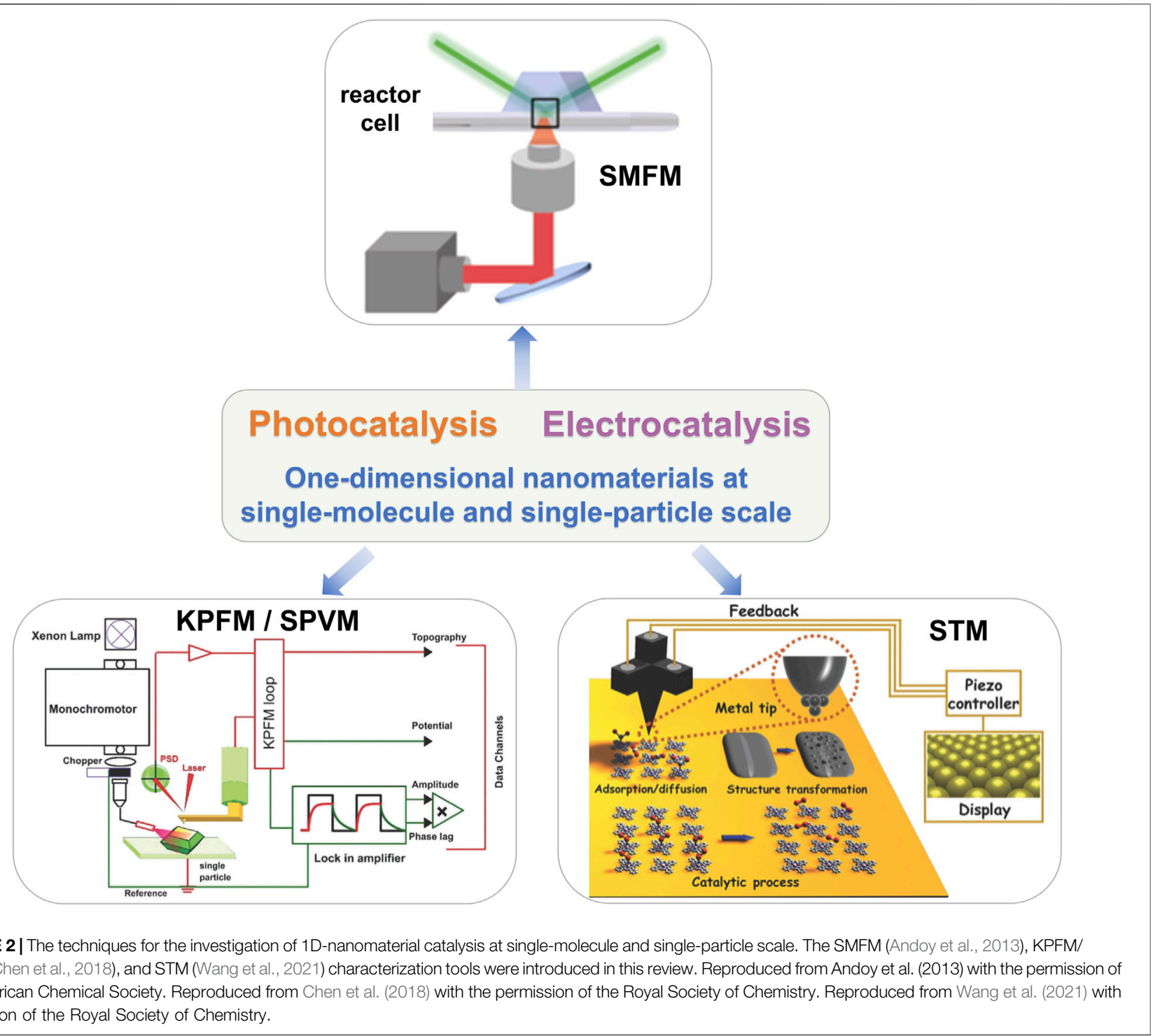

In this review, we summarized the recent progress of $1 \mathrm{D}$ nanomaterials by focusing on the applications in photocatalysis and electrocatalysis. We highlighted the advanced characterization techniques, including STM, AFM, SPVM, SMFM, and a variety of combined characterization methods, which have been used to study the catalytic mechanism of 1D nanomaterials. The 1D-nanostructured materials that can be applied to catalysis in the form of single molecule and single particle were summarized as three types: 1) monitoring of catalytic reactions on individual particles using the SMFM technique; 2) visualization of photogenerated charge distribution of a single particle using the kelvin probe force microscopy (KPFM)/SPVM technique; and 3) morphological analysis of single particles and single atoms using STM and AFM techniques. Finally, the challenges and future directions of the research on the catalytic mechanism of single-particle 1D nanomaterials have been prospected. To our best knowledge, there is no review on the application of single-particle or single-molecule characterization technology to 1D nanomaterial catalysis at present. This review provides a systematic introduction to this frontier field and opens the way for $1 \mathrm{D}$ nanomaterial catalysis.

\section{Photocatalysis}

Among nanomaterials, 1D-nanostructured materials with controllable morphology, large surface area, and more exposed active sites have attracted wide attention in photocatalysis. As a typical photocatalyst, the $\mathrm{TiO}_{2}$-nanostructured catalyst was a wide-gap semiconductor with excellent physical and chemical properties (Choi et al., 2010; Cao et al., 2018). The $1 \mathrm{D} \mathrm{TiO}_{2}$ nanofiber structure photocatalyst prepared through the foamassisted electrospinning method by $\mathrm{Wu}$ had a complete mesoporous channel and uniform pore structure (Hou et al., 2014), which provided a large number of active sites for adsorption reactants and an efficient route for gas transport. 
Thus, it was favorable for the precipitation rate of photocatalytic hydrogen production. In addition, the $\mathrm{TiO}_{2}$ catalyst with a $1 \mathrm{D}$ nanofiber structure was more stable than commercial P25 products due to the nanofiber structure inhibiting the aggregation of nanomaterials and stabilizing its morphology. However, it was not well understood how to precisely locate the catalytic active site in $1 \mathrm{D}$-nanostructured catalysts and to what extent to drive charge separation in the photocatalytic reactions. The lack of understanding of these issues will become the bottleneck for further improvement of the photocatalytic efficiency. Therefore, the exploration of the $1 \mathrm{D}$ photocatalyst at single-particle and single-molecule scale is urgent for the investigation of microscopic mechanisms.

To solve these problems, it was important to directly image individual photocatalytic particles or photocatalyst interfaces. Super-resolution fluorescence microscopy techniques open up new prospects for visualization of individual catalytic events and localize the catalytic activity sites at the single nanocatalyst level (Roeffaers et al., 2006; Xu et al., 2008). The Au@mSiO ${ }_{2}$ materials formed by encapsulation of single gold nanorods in mesoporous silica shells were catalyzed for quantitative imaging using superresolution fluorescence microscopy techniques (Zhou et al., 2012). These nanorods scatter laser light and emit it, making them easy to identify under an optical microscope. The relationship between the fluorescence intensity of individual nanorods and the time trajectory showed stochastic intensity bursts over the constant nanorods. Each stochastic intensity burst was marked by the catalytic production of fluorescent molecules. Simultaneously, the fluorescent molecules can be located from the fluorescence image by Gaussian fitting. This technique can image a single molecule and locate the active site, allowing the researcher to discover the complex patterns of catalytic activity on individual nanorods. The nanorods with the same surface composition on both sides and ends should have the same catalytic activity in conventional view; however, the catalytic reaction rate in the same surface was not constant as revealed in this work and presented a gradient distribution along the center to both ends of the nanorods. This could be reasonably explained by the defect density distribution on the nanorod surfaces in crystal growth.

With the development of probe microscope techniques, the charge carriers can be visualized via the spatially resolved SPV technique. For example, the function and properties of $1 \mathrm{D} \mathrm{TiO}_{2}$ can be modified by gold nanoparticles (Au NPs) with SPR (Wang et al., 2017). The plasma water oxidation could be realized on the surface of $\mathrm{Au} / \mathrm{TiO}_{2}$ under the central wavelength of about 550-nm absorption light. In this process, there was an obvious ring at the interface between Au NPs and $\mathrm{TiO}_{2}$ in the SPVM technique, and the surface photovoltage (SPV) was increased at the interface through the detailed analysis. There were two possible reasons for this phenomenon. One was that the holes generated by plasma resonance tended to accumulate at the interface between $\mathrm{Au}$ NPs and $\mathrm{TiO}_{2}$, and the other was that the Schottky barrier at the interface promoted the transfer of hot electrons to $\mathrm{TiO}_{2}$ and prevented its recombination. This discovery not only provided clear evidence for the distribution of hot holes but also confirmed that the Schottky barrier played an important role in promoting charge separation and stabilizing hot holes.

The investigation of 1D photocatalysts on single-molecule and single-particle scale will be helpful to understand the microscopic reaction and the charge separation, which will provide inspiration for us to design efficient photocatalysts.

\section{Electrocatalysis}

Noble metal catalysts have attracted wide attention for their excellent catalytic performance, but the high price and limited reserves prevent their large-scale use. In the present studies, researchers reduced or avoided the use of noble metals through loading and alloying strategies or development of non-noble metal catalysts (Seh et al., 2017). Gao et al. chemically synthesized $\mathrm{Pd} / \mathrm{CeO}_{2} / \mathrm{C} 1 \mathrm{D}$ nanostructure hybrid materials by the hydrothermal method (Gao et al., 2018). Compared to pure $\mathrm{Pd} / \mathrm{C}, \mathrm{CeO}_{2} / \mathrm{C}$, and a physical mixture of $\mathrm{Pd} / \mathrm{C}$ and $\mathrm{CeO}_{2} / \mathrm{C}$, the chemically synthesized $\mathrm{Pd} / \mathrm{CeO}_{2} / \mathrm{C} 1 \mathrm{D}$ nanostructure showed excellent catalytic activity and stability in the electrocatalytic hydrogen evolution reaction (HER). The main reason was that the chemical synthesis method had stronger interface coupling ability between $\mathrm{Pd}$ and $\mathrm{CeO}_{2}$ than the physical mixture method. However, this work did not explore the properties of the electrocatalyst interface at the single-particle scale, and the catalytic reaction mechanism was a little vague.

To further distinguish the reactivity of different catalyst surfaces and sites, the most direct method is to detect catalytic reactions in situ with spatial resolution on a single catalyst particle. The super-resolution imaging technique, such as STM, AFM, and SMFM, has gained considerable recognition at the level of a single molecule/particle, because of their ability to reveal and detect the dynamic system, providing detailed information about the individual molecule reaction, adsorption, and desorption process, as well as the distribution of active sites of single-particle catalysis (Carbonio et al., 2014). Other atom-resolved microscopes with distinctive features were also used in electrocatalytic reaction. AFM can provide morphological information of conductive and non-conductive samples. The degradation process of the $\mathrm{Pt}-\mathrm{Ni}$ alloy catalyst was studied by in situ electrochemical atomic force microscopy (ECAFM) during the accelerated durability test (ADT), and the coarsening process of the catalyst surface was observed during potential cycles (Khalakhan et al., 2017).

Among the non-noble metal electrocatalysts, carbon nanotubes are the most famous type of $1 \mathrm{D}$ nanomaterials. The SMFM imaging technique is also one of the advanced methods to characterize single molecules (Platnich et al., 2019; Bacic et al., 2020; Hao et al., 2020). Xu et al. studied the electrocatalytic fluorescence reaction by purified single-walled carbon nanotubes (SWNTs) using the SMFM imaging technique at the singlemolecule level (Xu et al., 2009). In the electrocatalytic fluorescence reaction, the SMFM imaging technique had the ability of super-resolution localization of single fluorescence products and of determining the catalytic active sites on SWNTs, indicating that the fluorescence bursts of individual products were attributed to the electrocatalytic reduction of the reactants on SWNTs. 
In short, the introduction of advanced characterization techniques plays an important role in revealing the reaction mechanism and determining the catalytic active sites. It also provides a powerful path for the design of novel electrocatalytic materials in the future.

\section{CONCLUSION AND PERSPECTIVES}

In this review, recent progress of $1 \mathrm{D}$ nanomaterials on the applications in photocatalysis and electrocatalysis was summarized. The single-molecule and single-particle investigation of $1 \mathrm{D}$ nanomaterials was reviewed, which had proved to be capable of improving the understanding of complex processes in the field of catalysis and gave deep insights into optimizing the performance of catalytic systems. This review provides a systematic introduction to the frontier field and opens the way for 1D nanomaterial catalysis.

To data, the properties of photocatalytic or electrocatalytic process are obtained from one individual particle. However, the preparation of single-particle nanomaterials still faces significant challenges due to the subtle effects of size, shape, and structural defects. At the same time, the present characterization technique is also very dependent on the external environment, which often causes randomness and volatility of data at the single-molecule

\section{REFERENCES}

Andoy, N. M., Zhou, X., Choudhary, E., Shen, H., Liu, G., and Chen, P. (2013). Single-Molecule Catalysis Mapping Quantifies Site-Specific Activity and Uncovers Radial Activity Gradient on Single 2D Nanocrystals. J. Am. Chem. Soc. 135 (5), 1845-1852. doi:10.1021/ja309948y

Bacic, L., Sabantsev, A., and Deindl, S. (2020). Recent Advances in Single-Molecule Fluorescence Microscopy Render Structural Biology Dynamic. Curr. Opin. Struct. Biol. 65, 61-68. doi:10.1016/j.sbi.2020.05.006

Byrappa, K., and Adschiri, T. (2007). Hydrothermal Technology for Nanotechnology. Prog. Cryst. Growth Characterization Mater. 53 (2), 117-166. doi:10.1016/j.pcrysgrow.2007.04.001

Cao, S., Zhang, S., Zhang, T., Fisher, A., and Lee, J. Y. (2018). Metal-Doped TiO2 Colloidal Nanocrystals With Broadly Tunable Plasmon Resonance Absorption. J. Mater. Chem. C. 6 (15), 4007-4014. doi:10.1039/C8TC00185E

Cao, S., Zhang, S., Zhang, T., Yao, Q., and Lee, J. Y. (2019). A Visible Light-NearInfrared Dual-Band Smart Window With Internal Energy Storage. Joule. 3 (4), 1152-1162. doi:10.1016/j.joule.2018.12.010

Carbonio, E. A., Prieto, M. J., Siervo, A. d., and Landers, R. (2014). From 1D to 3D $\mathrm{Ru}$ Nanostructures on a Pt Stepped Surface as Model Systems in Electrocatalysis: UHV-STM and XPS Study. J. Phys. Chem. C. 118 (49), 28679-28688. doi:10.1021/jp509574s

Chao, Y., Zhang, W., Zhou, P., Chen, H., Lu, S., Li, M., et al. (2021). An In-Situ NH4+-etched Strategy for Anchoring Atomic Mo Site on ZnIn2S4 Hierarchical Nanotubes for superior Hydrogen Photocatalysis. Sci. China Chem. 64 (10), 1716-1722. doi:10.1007/s11426-021-1063-2

Chen, R., Fan, F., Dittrich, T., and Li, C. (2018). Imaging Photogenerated Charge Carriers on Surfaces and Interfaces of Photocatalysts With Surface Photovoltage Microscopy. Chem. Soc. Rev. 47 (22), 8238-8262. doi:10.1039/ C8CS00320C

Choi, S. K., Kim, S., Lim, S. K., and Park, H. (2010). Photocatalytic Comparison of TiO2 Nanoparticles and Electrospun TiO2 Nanofibers: Effects of Mesoporosity and Interparticle Charge Transfer. J. Phys. Chem. C. 114 (39), 16475-16480. doi:10.1021/jp104317x and single-particle scale. For future, the first step is to improve the preparation methods of single-particle nanomaterials. Secondly, it is necessary to optimize the stability of advanced characterization techniques and unify standardized measurement. The results obtained from different research groups may be affected by the instrument settings and measurement environments. Finally, to enhance the efficiency of testing, high-speed acquisition and automated data analysis are required, and mathematical tools such as big data processing and machine learning will shine in these fields.

\section{AUTHOR CONTRIBUTIONS}

TG wrote the manuscript. PD revised the manuscript. QZ and SY conceived the structure of the manuscript. All the authors read and approved the manuscript.

\section{FUNDING}

The authors thank the National Natural Science Foundation of China (21805191), the Guangdong Basic and Applied Basic Research Foundation (2020A1515010982), and the Shenzhen Stable Support Project (20200812122947002).

Gao, T., Yang, J., Nishijima, M., Miller, H. A., Vizza, F., Gu, H., et al. (2018). Evidence of the Strong Metal Support Interaction in a Palladium-Ceria Hybrid Electrocatalyst for Enhancement of the Hydrogen Evolution Reaction. J. Electrochem. Soc. 165, F1147-F1153. doi:10.1149/2.0351814jes

George, S. M. (2010). Atomic Layer Deposition: An Overview. Chem. Rev. 110 (1), 111-131. doi:10.1021/cr900056b

Ghahremaninezhad, A., and Dolati, A. (2009). A Study on Electrochemical Growth Behavior of the Co-Ni alloy Nanowires in Anodic Aluminum Oxide Template. J. Alloys Compounds. 480 (2), 275-278. doi:10.1016/j.jallcom.2009.02.020

Hao, R., Peng, Z., and Zhang, B. (2020). Single-Molecule Fluorescence Microscopy for Probing the Electrochemical Interface. ACS Omega. 5 (1), 89-97. doi:10.1021/acsomega.9b03763

He, Y., He, Q., Wang, L., Zhu, C., Golani, P., Handoko, A. D., et al. (2019). SelfGating in Semiconductor Electrocatalysis. Nat. Mater. 18 (10), 1098-1104. doi:10.1038/s41563-019-0426-0

Hou, H., Shang, M., Gao, F., Wang, L., Liu, Q., Zheng, J., et al. (2016). Highly Efficient Photocatalytic Hydrogen Evolution in Ternary Hybrid $\mathrm{TiO} 2 / \mathrm{CuO} / \mathrm{Cu}$ Thoroughly Mesoporous Nanofibers. ACS Appl. Mater. Inter. 8 (31), 20128-20137. doi:10.1021/acsami.6b06644

Hou, H., Wang, L., Gao, F., Wei, G., Tang, B., Yang, W., et al. (2014). General Strategy for Fabricating Thoroughly Mesoporous Nanofibers. J. Am. Chem. Soc. 136 (48), 16716-16719. doi:10.1021/ja508840c

Khalakhan, I., Vorokhta, M., Kúš, P., Dopita, M., Václavů, M., Fiala, R., et al. (2017). In Situ probing of Magnetron Sputtered Pt-Ni alloy Fuel Cell Catalysts During Accelerated Durability Test Using EC-AFM. Electrochimica Acta. 245, 760-769. doi:10.1016/j.electacta.2017.05.202

Li, D., and Xia, Y. (2004). Direct Fabrication of Composite and Ceramic Hollow Nanofibers by Electrospinning. Nano Lett. 4 (5), 933-938. doi:10.1021/ nl049590f

Li, H., Wang, X., Xu, J., Zhang, Q., Bando, Y., Golberg, D., et al. (2013). OneDimensional CdS Nanostructures: A Promising Candidate for Optoelectronics. Adv. Mater. 25 (22), 3017-3037. doi:10.1002/adma.201300244

Linares, N., Silvestre-Albero, A. M., Serrano, E., Silvestre-Albero, J., and GarcíaMartínez, J. (2014). Mesoporous Materials for Clean Energy Technologies. Chem. Soc. Rev. 43 (22), 7681-7717. doi:10.1039/C3CS60435G 
Liu, G., Sun, C., Yang, H. G., Smith, S. C., Wang, L., Lu, G. Q., et al. (2010). Nanosized Anatase TiO2 Single Crystals for Enhanced Photocatalytic Activity. Chem. Commun. 46 (5), 755-757. doi:10.1039/B919895D

Liu, G., Wang, K., Gao, X., He, D., and Li, J. (2016a). Fabrication of Mesoporous NiFe2O4 Nanorods as Efficient Oxygen Evolution Catalyst for Water Splitting. Electrochimica Acta. 211, 871-878. doi:10.1016/j.electacta.2016.06.113

Liu, P., Zhao, Y., Qin, R., Mo, S., Chen, G., Gu, L., et al. (2016b). Photochemical Route for Synthesizing Atomically Dispersed Palladium Catalysts. Science. 352 (6287), 797-800. doi:10.1126/science.aaf5251

Low, J., Yu, J., Jaroniec, M., Wageh, S., and Al-Ghamdi, A. A. (2017). Heterojunction Photocatalysts. Adv. Mater. 29 (20), 1601694. doi:10.1002/ adma.201601694

Mai, L., Tian, X., Xu, X., Chang, L., and Xu, L. (2014). Nanowire Electrodes for Electrochemical Energy Storage Devices. Chem. Rev. 114 (23), 11828-11862. doi: $10.1021 /$ cr500177a

Novo, C., Funston, A. M., and Mulvaney, P. (2008). Direct Observation of Chemical Reactions on Single Gold Nanocrystals Using Surface Plasmon Spectroscopy. Nat. Nanotech. 3 (10), 598-602. doi:10.1038/nnano.2008.246

Patil, J. V., Mali, S. S., Kamble, A. S., Hong, C. K., Kim, J. H., and Patil, P. S. (2017). Electrospinning: A Versatile Technique for Making of 1D Growth of Nanostructured Nanofibers and its Applications: An Experimental Approach. Appl. Surf. Sci. 423, 641-674. doi:10.1016/j.apsusc.2017.06.116

Platnich, C. M., Hariri, A. A., Sleiman, H. F., and Cosa, G. (2019). Advancing Wireframe DNA Nanostructures Using Single-Molecule Fluorescence Microscopy Techniques. Acc. Chem. Res. 52 (11), 3199-3210. doi:10.1021/ acs.accounts.9b00424

Roeffaers, M. B. J., Sels, B. F., Uji-i, H., De Schryver, F. C., Jacobs, P. A., De Vos, D. E., et al. (2006). Spatially Resolved Observation of Crystal-Face-Dependent Catalysis by Single Turnover Counting. Nature. 439 (7076), 572-575. doi:10.1038/nature04502

Seh, Z. W., Kibsgaard, J., Dickens, C. F., Chorkendorff, I., Nørskov, J. K., and Jaramillo, T. F. (2017). Combining Theory and Experiment in Electrocatalysis: Insights Into Materials Design. Science. 355 (6321), eaad4998. doi:10.1126/science.aad4998

Sui, R., and Charpentier, P. (2012). Synthesis of Metal Oxide Nanostructures by Direct Sol-Gel Chemistry in Supercritical Fluids. Chem. Rev. 112 (6), 3057-3082. doi:10.1021/cr2000465

Sun, Y., Wang, W., Qin, J., Zhao, D., Mao, B., Xiao, Y., et al. (2016). Oxygen Vacancy-Rich Mesoporous W18O49 Nanobelts With Ultrahigh Initial Coulombic Efficiency Toward High-Performance Lithium Storage. Electrochimica Acta. 187, 329-339. doi:10.1016/j.electacta.2015.11.064

Tachikawa, T., Yamashita, S., and Majima, T. (2011). Evidence for Crystal-FaceDependent TiO2 Photocatalysis From Single-Molecule Imaging and Kinetic Analysis. J. Am. Chem. Soc. 133 (18), 7197-7204. doi:10.1021/ja201415j

Tang, M. L., Liu, N., Dionne, J. A., and Alivisatos, A. P. (2011). Observations of Shape-Dependent Hydrogen Uptake Trajectories From Single Nanocrystals. J. Am. Chem. Soc. 133 (34), 13220-13223. doi:10.1021/ja203215b

Tian, B., Zheng, X., Kempa, T. J., Fang, Y., Yu, N., Yu, G., et al. (2007). Coaxial Silicon Nanowires as Solar Cells and Nanoelectronic Power Sources. Nature. 449 (7164), 885-889. doi:10.1038/nature06181

Wang, H., Zhang, L., Chen, Z., Hu, J., Li, S., Wang, Z., et al. (2014). Semiconductor Heterojunction Photocatalysts: Design, Construction, and Photocatalytic Performances. Chem. Soc. Rev. 43 (15), 5234-5244. doi:10.1039/C4CS00126E

Wang, S., Gao, Y., Miao, S., Liu, T., Mu, L., Li, R., et al. (2017). Positioning the Water Oxidation Reaction Sites in Plasmonic Photocatalysts. J. Am. Chem. Soc. 139 (34), 11771-11778. doi:10.1021/jacs.7b04470
Wang, X., Wang, Y.-Q., Feng, Y.-C., Wang, D., and Wan, L.-J. (2021). Insights Into Electrocatalysis by Scanning Tunnelling Microscopy. Chem. Soc. Rev. 50 (10), 5832-5849. doi:10.1039/D0CS01078B

Xia, Y., Yang, P., Sun, Y., Wu, Y., Mayers, B., Gates, B., et al. (2003). OneDimensional Nanostructures: Synthesis, Characterization, and Applications. Adv. Mater. 15 (5), 353-389. doi:10.1002/adma.200390087

$\mathrm{Xu}$, W., Kong, J. S., Yeh, Y.-T. E., and Chen, P. (2008). Single-Molecule Nanocatalysis Reveals Heterogeneous Reaction Pathways and Catalytic Dynamics. Nat. Mater. 7 (12), 992-996. doi:10.1038/nmat2319

Xu, W., Shen, H., Kim, Y. J., Zhou, X., Liu, G., Park, J., et al. (2009). Single-Molecule Electrocatalysis by Single-Walled Carbon Nanotubes. Nano Lett. 9 (12), 3968-3973. doi:10.1021/nl900988f

Yuán, S., Liu, S., Zhang, Q., Zhang, M., Xu, B., and Ohno, T. (2018). Effects of the Atmosphere in a Hydrothermal Process on the Morphology and Photocatalytic Activity of Cerium Oxide. Chemcatchem. 10 (19), 4269-4273. doi:10.1002/ cctc. 201800659

Yuán, S., Xu, B., Zhang, Q., Liu, S., Xie, J., Zhang, M., et al. (2018). Development of the Visible-Light Response of CeO 2- X With a High Ce 3+ Content and its Photocatalytic Properties. ChemCatChem. 10 (6), 1267-1271. doi:10.1002/ cctc. 201701767

Yuan, S., Zhang, Q., Xu, B., Jin, Z., Zhang, Y., Yang, Y., et al. (2014). Porous Cerium Dioxide Hollow Spheres and Their Photocatalytic Performance. RSC Adv. 4 (107), 62255-62261. doi:10.1039/c4ra12127a

Yuan, S., Zhang, Q., Xu, B., Liu, S., Wang, J., Xie, J., et al. (2017). A New Precursor to Synthesize G-C3N4 With superior Visible Light Absorption for Photocatalytic Application. Catal. Sci. Technol. 7 (9), 1826-1830. doi:10.1039/c7cy00213k

Zhang, Q., Yuan, S., Xu, B., Xu, Y., Cao, K., Jin, Z., et al. (2018). A Facile Approach to Build Bi2O2CO3/PCN Nanohybrid Photocatalysts for Gaseous Acetaldehyde Efficient Removal. Catal. Today. 315, 184-193. doi:10.1016/ j.cattod.2018.03.071

Zhou, X., Andoy, N. M., Liu, G., Choudhary, E., Han, K.-S., Shen, H., et al. (2012). Quantitative Super-Resolution Imaging Uncovers Reactivity Patterns on Single Nanocatalysts. Nat. Nanotech. 7 (4), 237-241. doi:10.1038/nnano.2012.18

Zhu, H., Yuan, X., Yao, Q., and Xie, J. (2021). Shining Photocatalysis by GoldBased Nanomaterials. Nano Energy. 88, 106306. doi:10.1016/ j.nanoen.2021.106306

Conflict of Interest: The authors declare that the research was conducted in the absence of any commercial or financial relationships that could be construed as a potential conflict of interest.

Publisher's Note: All claims expressed in this article are solely those of the authors and do not necessarily represent those of their affiliated organizations, or those of the publisher, the editors, and the reviewers. Any product that may be evaluated in this article, or claim that may be made by its manufacturer, is not guaranteed or endorsed by the publisher.

Copyright (c) 2021 Yuan and Zhang. This is an open-access article distributed under the terms of the Creative Commons Attribution License (CC BY). The use, distribution or reproduction in other forums is permitted, provided the original author(s) and the copyright owner(s) are credited and that the original publication in this journal is cited, in accordance with accepted academic practice. No use, distribution or reproduction is permitted which does not comply with these terms. 